Provided for non-commercial research and education use. Not for reproduction, distribution or commercial use.

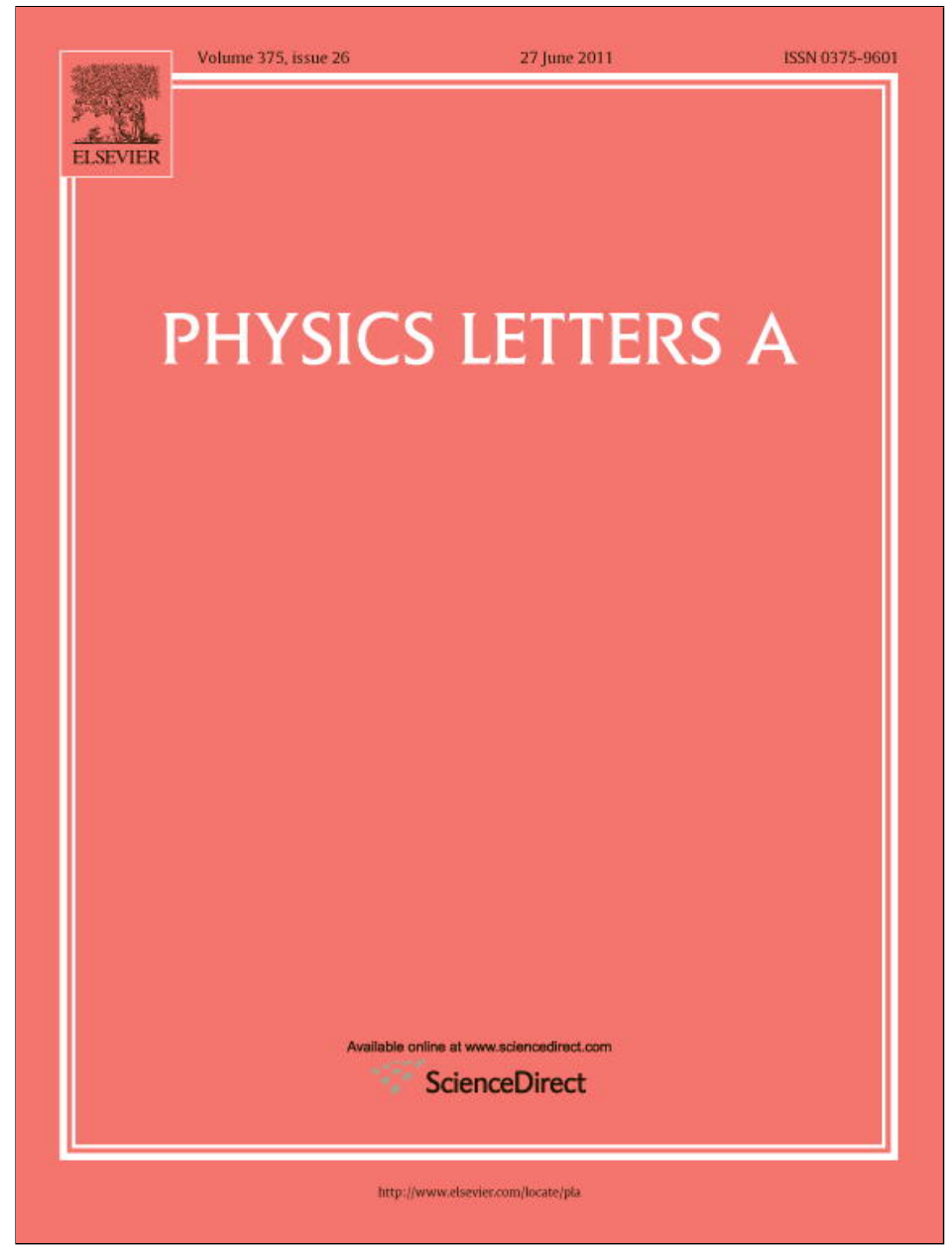

This article appeared in a journal published by Elsevier. The attached copy is furnished to the author for internal non-commercial research and education use, including for instruction at the authors institution and sharing with colleagues.

Other uses, including reproduction and distribution, or selling or licensing copies, or posting to personal, institutional or third party websites are prohibited.

In most cases authors are permitted to post their version of the article (e.g. in Word or Tex form) to their personal website or institutional repository. Authors requiring further information regarding Elsevier's archiving and manuscript policies are encouraged to visit:

http://www.elsevier.com/copyright 


\title{
Regular perturbation theory for two-electron atoms
}

\author{
I.D. Feranchuk*, V.V. Triguk \\ Belorussian State University, 4 Nezavisimosty Ave., 220030 Minsk, Belarus
}

\section{A R T I C L E I N F O}

\section{Article history:}

Received 23 March 2011

Received in revised form 13 May 2011

Accepted 16 May 2011

Available online 25 May 2011

Communicated by P.R. Holland

\section{Keywords:}

Two-particle Coulomb Green's function

Summation intermediate states

Two-electron atom

\begin{abstract}
A B S T R A C $T$
Regular perturbation theory (RPT) for the ground and excited states of two-electron atoms or ions is developed. It is shown for the first time that summation of the matrix elements from the electronelectron interaction operator over all intermediate states can be calculated in a closed form by means of the two-particle Coulomb Green's function constructed in the Letter. It is shown that the second order approximation of RPT includes the main part of the correlation energy both for the ground and excited states. This approach can be also useful for description of two-electron atoms in external fields.
\end{abstract}

(C) 2011 Elsevier B.V. All rights reserved.

\section{Introduction}

In spite of the long history of the two-electron atom in nonrelativistic quantum mechanics the problem of developing the most effective and universal method for the solution of the Schrödinger equation for such systems is still active (for example, [1]). This problem is of interest because it could be generalized as well for multi-electron atoms because of the two-particle character of the Coulomb potential.

At present the best accuracy for calculating the energy levels of two-electron atoms is achieved by means of the variational approach based on the special form of a many parameter trial wave function (for example, [2]). However, this method is difficult to apply for multi-electron atoms. Therefore a less accurate variational approach with a trial wave function in the form of a product of the one particle wave functions, the Hartree-Fock (HF) method, is commonly used in this case (cf., [3]). For example, the energy for the He atom (the $1^{1} S$ ground state) calculated numerically via the $\mathrm{HF}$ method is $E_{0}^{H F}=-2.86168$ [3], which differs from the more accurate variational result of $E_{0}^{R}=-2.90372$ [2] on the value of the correlation energy $\Delta E_{c}=E_{0}^{R}-E_{0}^{H F}=-0.04204$ (the Coulomb system of units is used in this Letter). At the same time a very simple analytical approximation of the Coulomb potential with an equal effective charge for each electron leads to $E_{0}^{e f f}=-2.84766$ [4]. This means that the correlation energy essentially is more important than the effect of the self-consistent field $\Delta E_{s c f}=E_{0}^{H F}-E_{0}^{\text {eff }}=-0.01402$ taken into account in the HF approximation.

\footnotetext{
* Corresponding author

E-mail address: fer@open.by (I.D. Feranchuk).
}

One can suppose that regular perturbation theory (RPT) on the basis of the independent Coulomb wave functions for each electron will prove to be very effective for taking into account the electron correlation. However, the realization of this approach is connected with the calculation of the slowly convergent sums of the twoparticle potential matrix elements over the whole spectrum of the intermediate states. Therefore at present only the variational perturbation theory is used for the estimation of these sums by means of a trial function [5]. Unfortunately it is also difficult to generalize this method for multi-electron atoms.

In the present Letter it is shown for the first time that the sum over the intermediate states in the second order RPT can be calculated in closed form through the two-particle Coulomb Green's function represented below analytically. This method is applied to both the ground and excited states of the two-electron atom. It is shown that the larger part of the correlation energy proves to be taken into account just in the second order approximation of RPT. Actually our purpose is not only the two-electron atom itself but also development of the approach that could be generalized for calculation of the correlation energy in multi-electron atoms and their interaction with external fields. This approach is expected to be effective because the RPT zeroth order approximation allowed us to calculate characteristics of the multi-electron atoms with rather good accuracy [6].

It should be also noted that the eigenfunction decomposition of the two-particle Coulomb Green's function was used earlier in the paper [7] where the relativistic QED-perturbation theory for two-electron atoms has been considered in details. However the sums over the intermediate states were calculated by means of the numerical solutions of the single-particle Dirac equation instead of the convolution of the analytical one-particle Coulomb Green's functions as it will be considered below in the present Letter. 


\section{Ground state energy}

Let us consider the application of RPT to the two-electron atom with the following Hamiltonian:

$\hat{H}=\sum_{i=1,2}\left[-\frac{\Delta_{i}}{2}-\frac{Z}{r_{i}}\right]+\frac{1}{\left|\vec{r}_{1}-\vec{r}_{2}\right|}$.

Potentials with effective charge $Z_{e}$ can be used in order to separate the zeroth-order Hamiltonian:

$\hat{H}_{0}=\frac{\hat{p}_{1}^{2}}{2}-\frac{Z_{e}}{r_{1}}+\frac{\hat{p}_{2}^{2}}{2}-\frac{Z_{e}}{r_{2}} ;$

$E_{m n}^{(0)}=-\frac{Z_{e}^{2}}{2}\left[\frac{1}{(n+1)^{2}}+\frac{1}{(m+1)^{2}}\right], \quad n, m=0,1,2, \ldots$

and the perturbation operator

$\hat{V}=-\frac{Z-Z_{e}}{r_{1}}-\frac{Z-Z_{e}}{r_{2}}+\frac{1}{\left|\vec{r}_{1}-\vec{r}_{2}\right|}$.

If the ground state is considered, the results for the energy in the zeroth and first-order approximations are well known:

$E_{0}^{(0)}=-Z_{e}^{2} ; \quad E_{0}^{(1)}=-2 Z_{e}\left(Z-Z_{e}\right)+\frac{5}{8} Z_{e}$.

Let us consider the second-order correction for the same state,

$E_{0}^{(2)}=\sum_{\lambda=0} \sum_{\mu=0}^{\prime} \frac{|\langle\lambda \mu|\hat{V}| 00\rangle|^{2}}{-Z_{e}^{2}-E_{\lambda}^{(0)}-E_{\mu}^{(0)}}$.

Here $\lambda, \mu$ are the set of quantum numbers for electrons in the intermediate states defined by the vectors $|\lambda \mu\rangle$ that are represented as the product of the Coulomb wave functions including the continuous spectrum. The term corresponding to the ground state $|00\rangle=\psi_{0}\left(\vec{r}_{1}\right) \psi_{0}\left(\vec{r}_{2}\right)$ should be omitted in the sum.

Expression (5) can be divided into two terms:

$$
\begin{aligned}
E_{0}^{(2)} & =2 \sum_{\lambda \neq 0} \frac{|\langle\lambda 0|\hat{V}| 00\rangle|^{2}}{-\frac{Z_{e}^{2}}{2}-E_{\lambda}}+\sum_{\lambda \neq 0} \sum_{\mu \neq 0} \frac{|\langle\lambda \mu|\hat{V}| 00\rangle|^{2}}{-Z_{e}^{2}-E_{\lambda}^{(0)}-E_{\mu}^{(0)}} \\
& \equiv \Sigma_{1}+\Sigma_{2} .
\end{aligned}
$$

The first term can be calculated on the basis of the approach of [8] that was used in the problem of the interaction of hydrogen-like atoms with external fields. In this method, the sum over intermediate states is expressed analytically through the Coulomb Green's function (CGF). In this case it leads to the following result:

$$
\begin{aligned}
\Sigma_{1}= & -2 \int \psi_{0}^{*}\left(\vec{r}_{1}\right) \psi_{0}^{*}\left(\vec{r}_{2}\right) \hat{V}\left(\vec{r}_{1}, \vec{r}_{2}\right) \psi_{0}^{*}\left(\vec{r}_{2}\right) \tilde{G}_{\frac{-z_{e}^{2}}{2}}\left(\vec{r}_{1}, \vec{r}_{1}^{\prime}\right) \\
& \times \psi_{0}^{*}\left(\vec{r}_{2}^{\prime}\right) \hat{V}\left(\vec{r}_{1}^{\prime}, \vec{r}_{2}^{\prime}\right) \psi_{0}\left(\vec{r}_{1}^{\prime}\right) \psi_{0}\left(\vec{r}_{2}^{\prime}\right) d \vec{r}_{1} d \vec{r}_{2} d \vec{r}_{1}^{\prime} d \vec{r}_{2}^{\prime} .
\end{aligned}
$$

Here $\tilde{G}_{E}\left(\vec{r}_{1}, \vec{r}_{1}^{\prime}\right)$ is the reduced CGF

$\tilde{G}_{E}\left(\vec{r}_{1}, \vec{r}_{1}^{\prime}\right)=G_{E}\left(\vec{r}_{1}, \vec{r}_{1}^{\prime}\right)-\frac{\psi_{0}^{*}\left(\vec{r}_{1}\right) \psi_{0}\left(\vec{r}_{1}^{\prime}\right)}{E-E_{0}}$,

which was calculated analytically in [9]. The total CGF is defined as the sum of the series over the spherical harmonics $Y_{l, m_{l}}(\Omega)$ (for example, [10]),

$$
\begin{aligned}
& G_{E}\left(\vec{r}, \vec{r}^{\prime}\right)=\sum_{l, m_{l}} \frac{1}{r r^{\prime}} G_{E, l}\left(r, r^{\prime}\right) Y_{l, m_{l}}^{*}(\Omega) Y_{l, m_{l}}\left(\Omega^{\prime}\right) \\
& G_{E, l}\left(r, r^{\prime}\right)=\frac{v}{Z} \frac{\Gamma(l+1-v)}{\Gamma(2 l+2)} M_{v, l+\frac{1}{2}}\left(\frac{2 Z}{v} r_{<}\right) W_{v, l+\frac{1}{2}}\left(\frac{2 Z}{v} r_{>}\right),
\end{aligned}
$$

with $v=\frac{Z}{\sqrt{-2 E}} ; M, W$ are the Whittaker functions, $r_{<}$and $r_{>}$are the minimal and maximal values from $r$ and $r^{\prime}$ accordingly.

Taking into account that $\psi_{0}(\vec{r})=\psi_{0}^{*}(\vec{r})=R_{1,0}(r) Y_{0,0}(\theta, \phi)$ (here $R_{1,0}(r)=2 Z^{\frac{3}{2}} e^{-r Z}$ is the radial part, and $Y_{0,0}(\theta, \phi)=\frac{1}{\sqrt{4 \pi}}$ is the angular part), one finds as the result of integration over the angular variables

$$
\begin{aligned}
\Sigma_{1}= & -2 \int R_{1,0}\left(r_{1}\right) R_{1,0}^{2}\left(r_{2}\right) \hat{V}_{0}\left(r_{1}, r_{2}\right) \tilde{G}_{\frac{-Z_{e}^{2}}{2}, 0}\left(r_{1}, r_{1}^{\prime}\right) R_{1,0}\left(r_{1}^{\prime}\right) \\
& \times R_{1,0}^{2}\left(r_{2}^{\prime}\right) \hat{V}_{0}\left(r_{1}^{\prime}, r_{2}^{\prime}\right) d r_{1} d r_{2} d r_{1}^{\prime} d r_{2}^{\prime},
\end{aligned}
$$

with $V_{0}\left(r_{1}, r_{2}\right)=-\left(Z-Z_{e}\right)\left(\frac{1}{r_{1}}+\frac{1}{r_{2}}\right)+\frac{1}{r_{>}}$; and $r_{>}$is the maximal value from $r_{1}, r_{2}$.

In order to calculate the second term in Eq. (6) let us use the identity:

$\int_{-\infty}^{\infty} \frac{d \chi}{(a+i \chi)(b-i \chi)}=-\frac{2 \pi}{a+b}, \quad a<0, b<0$.

This allows us to separate the summations over the quantum numbers of different electrons in $\Sigma_{2}$ :

$-\int_{-\infty}^{\infty} \frac{d \chi}{2 \pi} \sum_{\lambda, \mu \neq 0} \frac{\langle\lambda \mu|\hat{V}| 00\rangle\langle 00|\hat{V}| \lambda \mu\rangle}{\left(-\frac{Z_{e}^{2}}{2}-E_{\lambda}^{(0)}+i \chi\right)\left(-\frac{Z_{e}^{2}}{2}-E_{\mu}^{(0)}-i \chi\right)}$.

Each of the summations can then be calculated by means of CGF's after their analytical continuations to the complex values $E=\frac{-Z_{e}^{2}}{2} \pm i \chi$ :

$$
\begin{aligned}
\Sigma_{2}= & -\int_{-\infty}^{\infty} \frac{d \chi}{2 \pi} \int \psi_{0}\left(\vec{r}_{1}\right) \psi_{0}\left(\vec{r}_{2}\right) \psi_{0}\left(\vec{r}_{1}^{\prime}\right) \psi_{0}\left(\vec{r}_{2}^{\prime}\right) \\
& \times \hat{V}\left(\vec{r}_{1}, \vec{r}_{2}\right) \tilde{G}_{\frac{-z_{e}^{2}}{2}+i \chi}\left(\vec{r}_{1}, \vec{r}_{1}^{\prime}\right) \tilde{G}_{\frac{-z_{e}^{2}}{2-i \chi}}\left(\vec{r}_{2}, \vec{r}_{2}^{\prime}\right) \\
& \times \hat{V}\left(\vec{r}_{1}^{\prime}, \vec{r}_{2}^{\prime}\right) d \vec{r}_{1} d \vec{r}_{2} d \vec{r}_{1}^{\prime} d \vec{r}_{2}^{\prime} .
\end{aligned}
$$

The function

$$
\begin{aligned}
& \sum_{\lambda, \mu \neq 0} \frac{|\lambda \mu\rangle\langle\lambda \mu|}{E-E_{\lambda}-E_{\mu}} \\
& =2 \tilde{G}_{\frac{E}{2}}\left(\vec{r}_{1}, \vec{r}_{1}^{\prime}\right) \psi_{0}\left(\vec{r}_{2}\right) \psi_{0}\left(\vec{r}_{2}^{\prime}\right) \\
& \quad-\frac{1}{2 \pi} \int_{-\infty}^{\infty} \tilde{G}_{\frac{E}{2}+i \chi}\left(\vec{r}_{1}, \vec{r}_{1}^{\prime}\right) \tilde{G}_{\frac{E}{2}-i \chi}\left(\vec{r}_{2}, \vec{r}_{2}^{\prime}\right) d \chi
\end{aligned}
$$

is the two-particle reduced CGF and

$\sum_{\lambda, \mu} \frac{|\lambda \mu\rangle\langle\lambda \mu|}{E-E_{\lambda}-E_{\mu}}=-\frac{1}{2 \pi} \int_{-\infty}^{\infty} G_{\frac{E}{2}+i \chi}\left(\vec{r}_{1}, \vec{r}_{1}^{\prime}\right) G_{\frac{E}{2}-i \chi}\left(\vec{r}_{2}, \vec{r}_{2}^{\prime}\right) d \chi$

is the total two-particle CGF that is the result of convolution of two one-particle CGF's [11]

The one-particle part $\frac{-\left(Z-Z_{e}\right)}{r_{1}}-\frac{\left(Z-Z_{e}\right)}{r_{2}}$ of the perturbation operator doesn't contribute to (13) because of the orthogonality of the wave functions. Then Eq. (13) has the following form after integration over the angular variables:

$$
\Sigma_{2}=-\sum_{l=0}^{\infty} \frac{1}{2 l+1} \int_{-\infty}^{\infty} \frac{d \chi}{2 \pi} \int_{0}^{\infty} d r_{1} d r_{2} d r_{1}^{\prime} d r_{2}^{\prime}
$$




$$
\begin{aligned}
& \times R_{1,0}\left(r_{1}\right) R_{1,0}\left(r_{2}\right) R_{1,0}\left(r_{1}^{\prime}\right) R_{1,0}\left(r_{2}^{\prime}\right) \frac{r_{<}^{l}}{r_{>}^{l+1}} \frac{r_{<}^{\prime l}}{r_{>}^{\prime l+1}} \\
& \times \tilde{G}_{\frac{-Z_{e}^{2}}{2}+i \chi, l}^{\prime}\left(r_{1}, r_{1}^{\prime}\right) \tilde{G}_{\frac{-Z_{e}^{2}}{2}-i \chi, l}^{\prime}\left(r_{2}, r_{2}^{\prime}\right) r_{1}^{2} r_{2}^{2} r_{1}^{\prime 2} r_{2}^{\prime 2} .
\end{aligned}
$$

Here the radial part of the reduced CGF is calculated as follows:

$\tilde{G}_{\frac{-z_{e}^{2}}{2} \pm i \chi, l}^{\prime}\left(r, r^{\prime}\right)= \begin{cases}G_{\frac{-z_{e}^{2}}{2} \pm i \chi, 0}\left(r, r^{\prime}\right) \pm r r^{\prime} \frac{\psi_{0}(r) \psi_{0}\left(r^{\prime}\right)}{i \chi}, & l=0, \\ G_{\frac{-z_{e}^{2}}{2} \pm i \chi, l}\left(r, r^{\prime}\right), & l>0 .\end{cases}$

The second order correction depends on the effective charge in an analytical way. The denominator in Eq. (5) can be represented in the form $E_{0}^{(0)}-E_{n}^{(0)}=Z_{e}^{2}\left(\epsilon_{0}-\epsilon_{n}\right)$, where $\epsilon_{0}$ and $\epsilon_{n}$ are the values corresponding to $Z_{e}=1$. The perturbation operator can be written in the form

$$
\begin{gathered}
\hat{V}\left(\vec{r}_{1}, \vec{r}_{2}\right)=-\left(Z-Z_{e}\right) V_{1}\left(r_{1}, r_{2}\right) V_{2}\left(\vec{r}_{1}, \vec{r}_{2}\right), \\
V_{1}=\left(\frac{1}{r_{1}}+1 / r_{2}\right), V_{2}=\frac{1}{\left|\vec{r}_{1}-\vec{r}_{2}\right|} .
\end{gathered}
$$

If the scale variables $\vec{x}_{1,2}=Z_{e} \vec{r}_{1,2}$ are used for integration in matrix elements, we find that

$E_{0}^{(2)}=-a\left(Z-Z_{e}\right)^{2}+b\left(Z-Z_{e}\right)-c$.

So, one should calculate numerically only the parameters $a, b, c$ which don't depend on $Z$ and $Z_{e}$. When calculating $a$ and $b$, the integration over $r_{2}$ and $r_{2}^{\prime}$ in Eq. (10) can be performed analytically and numerical integration is only necessary over two variables:

$$
\begin{aligned}
& a=-8 \int e^{-x_{1}-x_{1}^{\prime}} \tilde{G}_{-\frac{1}{2}, 0}\left(x_{1}, x_{1}^{\prime}\right) d x_{1} d x_{1}^{\prime} ; \\
& b= 16 \int e^{-x_{1}-3 x_{1}^{\prime}}\left(e^{2 x_{1}^{\prime}}-1-x_{1}^{\prime}\right) \tilde{G}_{-\frac{1}{2}, 0}\left(x_{1}, x_{1}^{\prime}\right) d x_{1} d x_{1}^{\prime} ; \\
& c=-8 \int\left(e^{2 x_{1}}-1-x_{1}\right)\left(e^{2 x_{1}^{\prime}}-1-x_{1}^{\prime}\right) e^{-3 x_{1}-3 x_{1}^{\prime}} \\
& \times \tilde{G}_{-\frac{1}{2}, 0}\left(x_{1}, x_{1}^{\prime}\right) d x_{1} d x_{1}^{\prime}-\frac{1}{2 \pi} \sum_{l=0}^{\infty} \frac{1}{2 l+1} \\
& \times \int_{-\infty}^{\infty} d \chi \int 16 e^{-x_{1}-x_{2}-x_{1}^{\prime}-x_{2}^{\prime}} \tilde{G}_{\frac{-1}{2}+i \chi, l}^{\prime}\left(x_{1}, x_{1}^{\prime}\right) \\
& \times \tilde{G}_{\frac{-1}{2}-i \chi, l}^{\prime}\left(x_{2}, x_{2}^{\prime}\right) \frac{x_{<}^{l}}{x_{>}^{l+1}} \frac{x_{<}^{\prime l}}{x_{>}^{\prime l+1}} x_{1} x_{2} x_{1}^{\prime} x_{2}^{\prime}\left\{d^{4} x\right\}, \\
&\left\{d^{4} x\right\}=d x_{1} d x_{2} d x_{1}^{\prime} d x_{2}^{\prime} .
\end{aligned}
$$

One finds $a=1$ and $b=\frac{5}{8}$ with an accuracy of $10^{-8}$. The most complicated task is the calculation of the expression in Eq. (16) that leads to the coefficient $c$ in Eq. (19). It was found that the sum over $l$ converges better than $\sum_{l=0}^{\infty} \frac{1}{l^{3}}$ and $c$ was calculated as 0.15759 with an accuracy $\sim 3 \cdot 10^{-5}$.

Thus, the first three terms of RPT lead to the following result for the ground state energy:

$E_{0}^{(0)}+E_{0}^{(1)}+E_{0}^{(2)}=-Z^{2}+\frac{5}{8} Z-0.15759$.

This doesn't depend on the effective charge and it coincides with the result of the variational PT with considerable accuracy [12]. In the case of helium this leads to

$E_{0}^{R P T} \approx-2.90759 ; \quad \Delta E=E_{0}^{R}-E_{0}^{R P T} \approx 0.00387$.

This means that RPT converges rather quickly and about $90 \%$ of the correlation energy is taken into account in the second order approximation.

\section{Energies of the excited states}

Let us now apply RPT to the low excited states $\left(2^{1} S\right.$ and $\left.2^{3} S\right)$ of He-like system with configuration $(1 s)^{1}(2 s)^{1}$. If the Hamiltonian $\hat{H}_{0}$ is chosen in the form (1) with the same effective charge for both electrons the degeneracy of states with permutation of electrons should be taken into account in the zeroth order approximation:

$\left|\psi_{ \pm}^{(0)}\right\rangle=\frac{1}{\sqrt{2}}\left[\psi_{0}\left(\vec{r}_{1}\right) \psi_{1}\left(\vec{r}_{2}\right) \pm \psi_{1}\left(\vec{r}_{1}\right) \psi_{0}\left(\vec{r}_{2}\right)\right]=\frac{1}{\sqrt{2}}[|01\rangle \pm|10\rangle]$,

where $\left|\psi_{ \pm}\right\rangle$are the para- and orthosymmetric states correspondingly.

Zeroth order approximations for both states are defined by the following formula that coincides with the result in [4]

$E_{ \pm}^{(0)}=-\frac{5}{8} Z_{ \pm}^{2} ; \quad Z_{ \pm}=Z-\frac{4}{5}(K \pm I) ;$
$Z_{+}=1.8145, \quad Z_{-}=1.8497$,
$E_{+}^{(0)}=-2.0578, \quad E_{-}^{(0)}=-2.1383$.

Here, the effective charges were calculated from the condition that the RPT first order correction $E_{ \pm}^{(1)}=\left\langle\psi_{ \pm}^{(0)}|\hat{V}| \psi_{ \pm}^{(0)}\right\rangle$ should be equal to zero [13] and the Coulomb integrals are calculated in the usual way:

$K=\int \frac{\psi_{0}^{*}\left(\vec{x}_{1}\right) \psi_{1}^{*}\left(\vec{x}_{2}\right) \psi_{0}\left(\vec{x}_{1}\right) \psi_{1}\left(\vec{x}_{2}\right) d \vec{x}_{1} d \vec{x}_{2}}{\left|\vec{x}_{2}-\vec{x}_{1}\right|}=\frac{17}{81}$
$I=\int \frac{\psi_{0}^{*}\left(\vec{x}_{1}\right) \psi_{1}^{*}\left(\vec{x}_{2}\right) \psi_{0}\left(\vec{x}_{2}\right) \psi_{1}\left(\vec{x}_{1}\right) d \vec{x}_{1} d \vec{x}_{2}}{\left|\vec{x}_{2}-\vec{x}_{1}\right|}=\frac{16}{729}$.

Following the same ideas as for the ground state, one can write the APT second order correction in the following form:

$E_{ \pm}^{(2)}=\left(Z-Z_{ \pm}\right)^{2} A+\left(Z-Z_{ \pm}\right)(B \pm \Delta B)+(C \pm \Delta C)$.

After integration over the angular variables, one has

$$
\begin{aligned}
A= & -\int\left[4 e^{-x_{1}-x_{1}^{\prime}} \tilde{G}_{-\frac{1}{2}, 0}\left(x_{1}, x_{1}^{\prime}\right)\right. \\
& \left.+\frac{1}{8} e^{-\frac{1}{2} x_{1}-\frac{1}{2} x_{1}^{\prime}}\left(x_{1}-2\right)\left(x_{1}^{\prime}-2\right) \tilde{G}_{-\frac{1}{8}, 0}\left(x_{1}, x_{1}^{\prime}\right)\right] d x_{1} d x_{1}^{\prime} ; \\
B= & \int e^{-x_{1}-2 x_{1}^{\prime}}\left(-8-8 e^{x_{1}^{\prime}}-6 x_{1}^{\prime}-2 x_{1}^{\prime 2}-x_{1}^{\prime 3}\right) \\
& \times \tilde{G}_{\frac{-1}{2}, 0}\left(x_{1}, x_{1}^{\prime}\right) d x_{1} d x_{1}^{\prime}+\frac{1}{4} \int e^{-\frac{1}{2} x_{2}-\frac{5}{2} x_{2}^{\prime}}\left(x_{2}-2\right) \\
& \times\left(x_{2}^{\prime}-2\right)\left(e^{2 x_{2}^{\prime}}-x_{2}^{\prime}-1\right) \tilde{G}_{\frac{-1}{8}, 0}\left(x_{2}, x_{2}^{\prime}\right) d x_{2} d x_{2}^{\prime} \\
\Delta B= & -\frac{4}{27} \int e^{-x_{1}-2 x_{1}^{\prime}}\left(3 x_{1}^{\prime 3}-4 x_{1}^{2}-4 x_{1}^{\prime}\right) \tilde{G}_{-1}, 0 \\
& \left.-\frac{4}{27} \int e_{1}, x_{1}^{\prime}\right) d x_{1} d x_{1}^{\prime} x_{2}-\frac{5}{2} x_{2}^{\prime}\left(x_{2}-2\right)\left(2 x_{2}^{\prime}+3 x_{2}^{\prime 2}\right) \\
& \times \tilde{G}_{-1}^{8}, 0 \\
C & \left.-C_{|00\rangle}-\int x_{2}, x_{2}^{\prime}\right) d x_{2} d x_{2}^{\prime} ; \\
\times & \left.\left(e^{2 x_{1}^{\prime}}-1-x_{1}^{\prime}\right)-\frac{32}{729} x_{1} x_{1}^{\prime}\left(2+3 x_{1}\right)\left(2+3 x_{1}^{\prime}\right)\right] \\
\times & \tilde{G}_{-\frac{1}{8}, 0}\left(x_{1}, x_{1}^{\prime}\right) d x_{1} d x_{1}^{\prime}-\frac{1}{2 \pi} \sum_{l=0}^{\infty} \frac{1}{2 l+1} \int_{-\infty}^{\infty} d x
\end{aligned}
$$




$$
\begin{aligned}
& \times \int \frac{1}{2} e^{-x_{1}-x_{1}^{\prime}-\frac{x_{2}}{2}-\frac{x_{2}^{\prime}}{2}}\left(x_{2}-2\right)\left(x_{2}^{\prime}-2\right) \frac{x_{<}^{l}}{x_{>}^{l+1}} \frac{x_{<}^{\prime l}}{x_{>}^{\prime l+1}} \\
& \times \tilde{G}_{-\frac{5}{16}+i \chi, l}^{\prime}\left(x_{1}, x_{1}^{\prime}\right) \tilde{G}_{-\frac{5}{16}-i \chi, l}^{\prime}\left(x_{2}, x_{2}^{\prime}\right) x_{1} x_{2} x_{1}^{\prime} x_{2}^{\prime}\left\{d^{4} x\right\} ; \\
& \Delta C=-C_{|00\rangle}-\frac{4}{27} \int e^{-\frac{5}{2} x_{1}-\frac{5}{2} x_{1}^{\prime}} \tilde{G}_{-\frac{1}{8}, 0}\left(x_{1}, x_{1}^{\prime}\right)\left(2 x_{1}^{\prime}+3 x_{1}^{\prime 2}\right) \\
& \times\left(e^{2 x_{1}}-x_{1}-1\right)\left(x_{1}-2\right) d x_{1} d x_{1}^{\prime}-\frac{1}{2 \pi} \sum_{l=0}^{\infty} \frac{1}{2 l+1} \int_{-\infty}^{\infty} d \chi \\
& \times \int \frac{1}{2} e^{-x_{1}-\frac{x_{1}^{\prime}}{2}-\frac{x_{2}}{2}-x_{2}^{\prime}}\left(x_{2}-2\right)\left(x_{1}^{\prime}-2\right) \frac{x_{<}^{l}}{x_{>}^{l+1}} \frac{x_{<}^{\prime l}}{x_{>}^{\prime l+1}} \\
& \times \tilde{G}_{-\frac{5}{16}+i \chi, l}^{\prime}\left(x_{1}, x_{1}^{\prime}\right) \tilde{G}_{-\frac{5}{16}-i \chi, l}^{\prime}\left(x_{2}, x_{2}^{\prime}\right) x_{1} x_{2} x_{1}^{\prime} x_{2}^{\prime}\left\{d^{4} x\right\} ; \\
& \tilde{G}_{\frac{-5}{16} \pm i \chi, l}^{\prime}\left(x, x^{\prime}\right)= \begin{cases}G_{\frac{-5}{16} \pm i \chi, 0}\left(x, x^{\prime}\right) \pm x x^{\prime} \frac{\psi_{0}(x) \psi_{0}\left(x^{\prime}\right)}{-\frac{3}{16} \mp i \chi}, & l=0, \\
G_{\frac{-5}{16} \pm i \chi, l}\left(x, x^{\prime}\right), & l>0 ;\end{cases} \\
& C_{|00\rangle}=\frac{8}{3}\left|\left\langle 00\left|\frac{1}{\left|\vec{x}_{2}-\vec{x}_{1}\right|}\right| 01\right\rangle\right|^{2} \text {. }
\end{aligned}
$$

After numerical integration we get $A \approx-\frac{5}{8}, B \approx \frac{17}{81}, \Delta B \approx \frac{16}{729}, C=$ -0.08095 , and $\Delta C=-0.03354$. Consequently, the second order RPT energy for these states can be written as

$E_{2^{1} S}=-\frac{5}{8} Z^{2}+\frac{169}{729}-0.11449$,

$E_{2^{3} S}=-\frac{5}{8} Z^{2}+\frac{137}{729}-0.04741$.

The dependence on the effective charge $Z_{ \pm}$vanishes in this case, just as for the ground state. For helium we get -2.1716 for the $2^{3} S$ state and -2.1508 for $2^{1} S$, which can be compared with the variational results [14] -2.175229 and -2.145973 . It means that the correlation energies for the excited states are taken into account with the same accuracy as for the ground state.

Eqs. (32), (33) can also be compared with the known [15] $Z^{-1}$ perturbation theory expansions:

$E_{2^{1} S}=-\frac{5}{8} Z^{2}+\frac{169}{729}-0.114510+\cdots$,

$E_{2^{3} S}=-\frac{5}{8} Z^{2}+\frac{137}{729}-0.047409-\cdots$.

\section{Concluding remarks}

So, the two-particle Coulomb Green's function is found in the Letter. It allows one to fulfil in the closed form the summation of transition matrix elements from the electron-electron interaction operator over all intermediate states and to develop RPT on this basis. Efficiency of this approach is analyzed when calculating the second order approximation for the energies of two-electron atoms. In this particular case the low level energies were calculated by other methods with essentially higher accuracy. Therefore applications of RPT for higher order approximations, multi-electron systems and analysis of the interaction between atom and external fields are more interesting. Let us consider briefly several examples in order to show qualitatively how to extend the method for these cases. We suppose to discuss corresponding numerical results in a separate paper.

If standard formulas of the perturbation theory [4] are used with the operator (3) the third order correction to the ground state energy can be represented in the form analogous to (19) with the same scaling transformation

$E_{0}^{(3)}=a_{1} Z_{e}+\left(b_{1}-2 a_{1} Z\right)+\frac{a_{1} Z^{2}-b_{1} Z-c_{1}}{Z_{e}}$.
Here the coefficients $a_{1}, b_{1}, c_{1}$ are expressed in a closed form by means of the two-particle Coulomb Green's functions with the effective charge $Z_{e}=1$ and should be calculated numerically. The value $E_{0}^{(3)}$ can be minimized relatively to $Z_{e}$ that leads to the following expression

$E_{0}^{(3)}=2 \sqrt{a_{1}^{2} Z^{2}-a_{1} b_{1} Z-a_{1} c_{1}}+b_{1}-2 a_{1} Z$.

It shows that the RPT successive terms are not reduced to the series over $Z^{-1}$ and it can be useful for analysis of the singularity of the function $E_{0}(Z)$ [5].

Hamiltonian of a three-electron atom can be divided on the zeroth order Hamiltonian $\hat{H}_{0}$ and the perturbation operator $\hat{V}$ by the following way

$\hat{H}_{0}=\sum_{i=1}^{3} \frac{\hat{p}_{i}^{2}}{2}-Z_{e}^{(1)}\left(\frac{1}{r_{1}}+\frac{1}{r_{2}}\right)-\frac{Z_{e}^{(2)}}{r_{3}} ;$

$\hat{V}=-\left(Z-Z_{e}^{(1)}\right)\left(\frac{1}{r_{1}}+\frac{1}{r_{2}}\right)-\frac{Z-Z_{e}^{(2)}}{r_{3}}+\sum_{i=1, j \neq i}^{3} \frac{1}{\left|\vec{r}_{i}-\vec{r}_{j}\right|}$,

with different effective charges $Z_{e}^{(1,2)}$ for internal and external shells. In the zeroth order approximation electrons are considered as distinguishable particles without taking into account the exchange corrections. It was shown recently [6] that this approach allows one to calculate physical characteristics of multi-electron atoms and ions with rather good accuracy if the effective charges are chosen in accordance with some analytical formula.

In order to take into account the permutation symmetry of the wave function let us use the projection operators [13]

$\hat{T}_{i j}^{(s)}=\frac{1}{\sqrt{2}}\left(1+s \hat{P}_{i j}\right), \quad s=(-1)^{S_{i j}}$,

where $\hat{P}_{i j}$ is the permutation operator and $S_{i j}$ is the spin of the electron pair.

The following equation for the wave function $\Psi_{0}\left(\vec{r}_{1}, \vec{r}_{2}, \vec{r}_{3}\right)$ of the three electron atom ground state can be considered instead of the Schrödinger equation [13]

$\left\{\hat{H}-E_{0}\right\}\left(1-P_{13}\right)\left(1+P_{23}\right) \psi_{0}\left(\vec{r}_{1}, \vec{r}_{2}, \vec{r}_{3}\right)=0 ;$

$\Psi_{0}\left(\vec{r}_{1}, \vec{r}_{2}, \vec{r}_{3}\right)=\frac{1}{2}\left(1-P_{13}\right)\left(1+P_{23}\right) \psi_{0}\left(\vec{r}_{1}, \vec{r}_{2}, \vec{r}_{3}\right)$.

The wave function $\psi_{0}\left(\vec{r}_{1}, \vec{r}_{2}, \vec{r}_{3}\right)$ is calculated by means of RPT with the transformed perturbation operator but without additional symmetrization

$\hat{V}_{R}\left(E_{0}\right)=\hat{V}(1+\hat{P})+\left(\hat{H}_{0}-E_{0}\right) \hat{P} ; \quad \hat{P}=P_{23}-P_{13}-P_{13} P_{23}$.

It can be shown that in this representation the exchange and correlation corrections are taken into account in the RPT series simultaneously and all sums over the intermediate states are reduced to the two-particle Coulomb Green's functions.

RPT can be also useful for analysis of the Rydberg states of helium in order to take into account the polarization effects [16]. In this case Hamiltonian of the system in the scaled variables has the following form [16]

$$
\begin{aligned}
& \hat{H}_{0}(r)=-\left(\nabla_{r}^{2}+\frac{4}{r}\right) ; \quad \hat{H}_{0}(x)=-\left(\nabla_{x}^{2}+\frac{2}{x}\right) ; \\
& \hat{V}(\vec{x}, \vec{r})=\frac{2}{|\vec{x}-\vec{r}|}-\frac{2}{x}-\frac{2 m}{M_{H e}+m} \vec{\nabla}_{r} \vec{\nabla}_{x} .
\end{aligned}
$$


In the RPT second order the energy shift $\Delta$ of the Rydberg level $E(1 s N L)$ is represented in a closed form in terms of the reduced Coulomb Green's functions

$$
\begin{aligned}
\Delta= & -\int_{-\infty}^{\infty} \frac{d \chi}{2 \pi} \int \psi_{0}(\vec{r}) \Psi_{N L M}^{*}(\vec{x}) \hat{V}(\vec{r}, \vec{x}) \tilde{G}_{-4+i \chi}\left(\vec{r}, \vec{r}^{\prime}\right) \\
& \times \tilde{G}_{\frac{-1}{N^{2}}-i \chi}\left(\vec{x}, \vec{x}^{\prime}\right) \hat{V}\left(\vec{r}^{\prime}, \vec{x}^{\prime}\right) \psi_{0}\left(\vec{r}^{\prime}\right) \Psi_{N L M}\left(\vec{r}_{2}^{\prime}\right) d \vec{r} d \vec{x} d \vec{r}^{\prime} d \vec{x}^{\prime} .
\end{aligned}
$$

It can be calculated numerically without the expansion of the integrand in a formal power series over $\frac{\hat{H}_{0}(x)}{\hat{H}_{0}(r)}$ as in the paper [16].

\section{References}

[1] P.E. Grabowski, Phys. Rev. A 81 (2010) 032508.
[2] K. Frankowski, C. Pekeris, Phys. Rev. 146 (1966) 145;

D. Freund, B. Huxtable, J. Morgan III, Phys. Rev. A 29 (1984) 980.

[3] F.C. Fischer, The Hartree-Fock Method for Atoms, Wiley, New York, 1997.

[4] L.D. Landau, E.M. Lifshitz, Quantum Mechanics, 3rd ed., Pergamon Press, Oxford, 1977.

[5] J. Zamastil, Z. Čížek, L. Scála, M. Šimánek, et al., Phys. Rev. A 81 (2010) 032118.

[6] V.V. Triguk, I.D. Feranchuk, J. Appl. Spectroscopy 77 (2011) 749.

[7] I. Lindgren, H. Persson, S. Salomonson, L. Labzowsky, Phys. Rev. A 181 (1995) 1167.

[8] A. Dalgarno, J.T. Lewis, Proc. Roy. Soc. 233A (1955) 70.

[9] L.C. Hostler, Phys. Rev. 178 (1969) 126.

[10] L.C. Hostler, J. Math. Phys. 5 (1964) 591

[11] R. Shakeshaft, Phys. Rev. A 70 (2004) 042704

12] C.W. Sherr, R.E. Knight, Rev. Mod. Phys. 35 (1963) 436

[13] I. Feranchuk, L. Komarov, I. Nichipor, A. Ulyanenkov, Ann. Phys. (NY) 238 (1995) 370.

14] C.L. Pekeris, Phys. Rev. 126 (1962) 1470.

[15] V. Yerokhin, K. Pachucki, Phys. Rev, A 81 (2010) 022507.

[16] R.J. Drachman, Phys. Rev. A 26 (1982) 1228. 